Western University

Scholarship@Western

Department of Economics Research Reports

Economics Working Papers Archive

1993

\title{
A Survey of Recent Empirical Work Concerning Auctions
}

Kenneth Hendricks

Harry J. Paarsch

Follow this and additional works at: https://ir.lib.uwo.ca/economicsresrpt

Part of the Economics Commons

Citation of this paper:

Hendricks, Kenneth, Harry J. Paarsch. "A Survey of Recent Empirical Work Concerning Auctions." Department of Economics Research Reports, 9309. London, ON: Department of Economics, University of Western Ontario (1993). 


\section{RESEARCH REPORT 9309}

A Survey of Recent Empirical Work Concerning Auctions

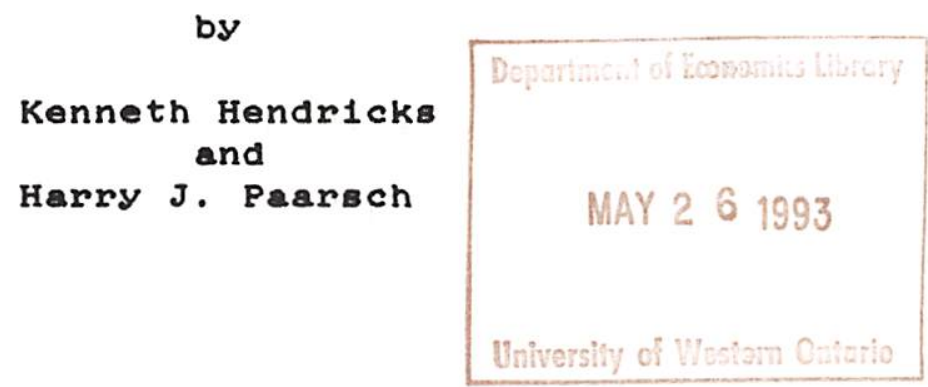

April 1993

\section{Department of Economics}

\section{Social Science Centre}

University of Western Ontar1o

London, Ontario, Canada

N6A $5 \mathrm{C} 2$ 


\title{
A SURVEY OF RECENT EMPIRICAL WORK CONCERNING AUCTIONS*
}

\author{
by \\ Kenneth Hendricks ${ }^{\dagger}$ \\ and \\ Harry J. Paarsch ${ }^{\ddagger}$
}

April 1993

* Both Hendricks and Paarsch are grateful to the SSHRC of Canada for financial support. We thank Chris Robinson for comments. Any errors are our own.

† Associate Professor, Department of Economics, University of British Columbia, Vancouver, British Columbia, Canada V6T 1 Z1.

$\ddagger$ Assistant Professor, Department of Economics, University of Western Ontario, London, Ontario, Canada N6A 5C2. 


\title{
A Survey of Recent Empirical Work concerning Auctions
}

\author{
Kenneth Hendricks \\ University of British Columbia, Vancouver, British Columbia, Canada, V6T 1Z1 \\ Harry J. Paarsch \\ University of Western Ontario, London, Ontario, Canada N6A 5C2
}

In this paper, we survey some recent empirical work concerning auctions. We outline two complementary approaches to the empirical analysis of auctions, and then discuss several recent developments in the econometric analysis of field data concerning auctions.

\section{Introduction}

The development of appropriate game-theoretic tools has made the study of auctions one of the most fruitful areas of theoretical research during the past decade; see Milgrom (1985, 1987) and McAfee and McMillan (1987) for surveys. Auctions are of special interest to economists because they are explicit mechanisms which describe how prices are formed. In addition, many transactions (particularly between the public and private sectors) involve auctions, so understanding how they work has practical value. Recently, a number of empirical researchers have used game-theoretic models of auctions to interpret actual field data from these institutions. In this paper, we discuss this research, focusing upon the problems encountered in taking the theory to data and the merits of proposed solutions.

The main components of an auction model consist of a set of potential buyers, the joint distribution of valuations for these potential buyers, and a reserve price rule used by the seller. Each potential buyer is assumed to know his valuation, but not those of his opponents. The probability law determining the valuations of potential buyers is assumed to be common knowledge. The auction rules determine what is bid and the pay-off to each potential buyer as a function of submitted bids. When formulated as a game of incomplete information, the Bayesian-Nash equilibrium of the model expresses each potential buyer's bid as a function of his valuation, the number of potential buyers, the reserve price, and the distribution of valuations of 
opponents conditional upon his own valuation. ${ }^{1}$ This in turn can be used to determine the distribution of the winning bid, the market price.

Recent empirical research has had two main goals: The first is to test the behavioural theory. Do potential buyers bid according to a Bayesian-Nash equilibrium? If the valuations of potential buyers and the probability law determining these valuations are known to the researcher, then this question is easily addressed by comparing the bids submitted with those predicted by the equilibrium bidding strategy. This approach is followed in experiments where researchers choose the probability law and draw the valuations for potential buyers. See, for example, the work of Cox, Roberson, and Smith (1982); Cox, Smith, and Walker (1985,1988); Dyer, Kagel, and Levin (1989); and Kagel and Levin (1986). Experimental studies suffer from one drawback: the experience of the subjects may be too limited and the compensation too small for them to perform the requisite optimizing calculations. This leaves a useful role for the careful study of field data. The difficulty with field data, however, is that neither the valuations of potential buyers nor the probability law determining these valuations is observed by the researcher.

The second goal of recent empirical research is to identify the probability law governing the valuations of potential buyers. This exercise is central to implementing optimal selling mechanisms. The literature on mechanism design has been criticized as lacking practical value because the optimal mechanism depends upon random variables whose distributions are typically unknown to the designer. In auctions, however, the equilibrium bid strategy of a potential buyer is usually an increasing function of his valuation. Consequently, if the researcher assumes that potential buyers bid according to Bayesian-Nash equilibrium strategies, then it may be possible to estimate the underlying probability law of valuations using bid data from a crosssection of auctions.

Hendricks and Porter, with a number of co-authors, have attempted to test the theory for U.S. federal auctions of offshore drainage leases. These are leases which are adjacent to tracts upon which an oil or a gas deposit (or both) has been discovered. Hendricks and Porter have provided evidence that firms owning the adjacent tracts, called "neighbour firms", are well informed concerning the value of the drainage

1 If the reserve price is unknown to a potential buyer at the time he chooses his bid, then the prior distribution of the reserve price conditional upon his valuation is what matters. 
lease and coordinate their bidding decisions. Non-neighbour firms are relatively uninformed. Under these conditions, the Bayesian-Nash equilibrium imposes restrictions upon the relationship between the distribution of the maximum non-neighbour bid and the distribution of the neighbour bid. Furthermore, these restrictions do not depend upon the functional form of the probability law for valuations. In the absence of unobserved heterogeneity across auctions, knowledge of the valuations or the probability law determining these valuations is unnecessary to test these restrictions. Because the Hendricks and Porter approach is non-structural, the presence of either observed or unobserved heterogeneity can complicate matter. It is possible that other models of behaviour may have the same reduced-form predictions that Hendricks and Porter et al. outline.

In contrast to the non-structural approach of Hendricks and Porter, Paarsch $(1989,1991,1992)$ has proposed using the entire structure of the game to derive the exact data generating process of equilibrium behaviour. This approach admits observed covariate heterogeneity in a straightforward and model consistent way, and can admit some forms of unobserved heterogeneity. The main difficulty with the structural approach is the complexity of the equilibrium bid functions which are typically highly non-linear. In some cases, the equilibrium bid functions have no closed-form solutions. The estimation procedures developed by Donald and Paarsch $(1991,1993 \mathrm{a})$ require that the joint distributions belong to particular families of distributions which admit numerical solutions to a closed-form solution to the bid function. The specification can then be estimated using non-linear programming techniques to obtain the maximum likelihood estimator. Alternatively, Laffont, Ossard, and Vuong (1991) have proposed a simulated non-linear least squares estimator which requires fewer restrictions upon the distribution of the latent heterogeneity. Nevertheless, a specific distributional assumption must still be made. More recently, Elyakime, Laffont, Loisel, and Vuong (forthcoming) have proposed non-parametric methods for estimating the probability law of valuations.

The parametric models provide a test of the joint hypothesis that the distribution of valuations belongs to the assumed family of distributions and that potential buyers bid according to a Bayesian-Nash equilibrium. In principle, it may be possible to distinguish between the behavioural assumption and functional form assumptions. For example, in his study of timber auctions in British Columbia Paarsch $(1989,1991)$ found that some regions used a first-price sealed-bid auction to sell cutting rights, 
while others used an open ascending (English) auction. Paarsch investigated whether the probability law determining valuations in these two types of auctions is the same. Under this hypothesis, if potential buyers are bidding in each type of auction according the Bayesian-Nash equilibrium, then the distributions of valuations that are estimated using bid data from English and first-price sealed-bid auctions should be identical.

This paper is organized as follows. In section 2, we discuss the non-structural (reduced-form) approach to testing the theory, while in section 3 we introduce the reader to structural econometric models of auctions within the independent private values paradigm by examining English auctions. Subsequently, in section 4 we examine several recent developments in the econometric analysis of field data concerning first-price sealed-bid auctions within the independent private values paradigm. In section 5 , we summarize the paper and conclude.

\section{Non-structural Approach}

Consider the following bidding environment. An indivisible object with unknown common value $V$ is to be sold at a first-price sealed-bid auction. The participants consist of an informed seller who observes a private signal and sets a positive reserve price $R$ before the bids are revealed, an informed buyer who observes a private signal $X$, and an arbitrary number of uninformed buyers who only observe a public signal $Z$. The signals lie in an $N$-dimensional space. The joint distribution of $(V, X, R)$ given $Z=z$ is common knowledge and denoted by $F(v, x, r ; z)$. Potential buyers submit sealed bids without knowing the reserve price. A bidder wins the object with certainty if his bid is strictly greater than any other bid and not less than the reserve price. If more than one bidder submits the same bid and those bids are greater than the reserve price, then the object is allocated according to some rationing rule that is independent of the reserve price. The winner of the auction receives the object in exchange for his bid.

This bidding environment is a good description of the sale of drainage leases by the U.S. government off the coasts of Louisiana and Texas. There $V$ represents the value of the unknown amounts of oil and gas that can be extracted from the drainage tract; $X$ represents geological information which neighbour firms obtain from drilling wells on their leases; and $Z$ represents the information which non-neighbour firms possess, seismic information and observable production on the adjacent tracts. 
Working with data from tracts sold during the period 1959 to 1979 , Hendricks and Porter (1988) established that neighbour firms were considerably better informed concerning the value of the drainage tracts than non-neighbour firms. They also provided evidence of collusion among the neighbour firms, suggesting that effectively there was only one informed potential buyer.

The pre-announced minimum (reserve) price for drainage leases was $\$ 25$ per acre. Most tracts were 2,250 acres, which implies that any bid less than $\$ 67,250$ was rejected. However, the government reserved the right to reject higher bids if it believed those bids were too low. The basis upon which the government made this judgement was its own private estimate of the value of the lease (obtained from the U.S. Geological Survey) and the number of actual bidders. Thus, from the perspective of the potential buyers the reserve price was effectively a random variable which was likely to be correlated with $(V, X)$. The highest bid was rejected on approximately 20 percent of the leases. Although the rejection decision was made after the bids were submitted, Hendricks, Porter, and Spady (1989) found no evidence of strategic behaviour upon the part of the government. The rejections occurred mostly on tracts where only one bid was submitted, usually by a neighbour firm, and where the value of that bid was low, less than $\$ 1$ million. Bids over $\$ 5$ million dollars were almost always accepted. Hendricks, Porter, and Spady concluded that

the purpose of the government's rejection policy was to reduce the incentive that firms might have had to bid the pre-announced minimum price on tracts that, on the basis of public information, were regarded as low value tracts.

Hendricks, Porter, and Wilson (1992) have analyzed first-price sealed-bid auctions with an informed potential buyer and a random reserve price. The key regularity condition which they imposed upon the joint distribution of $(V, X, R)$ is that these random variables be affiliated, a concept first introduced in the bidding literature by Milgrom and Weber (1982a). Roughly speaking, affiliation requires $(V, X, R)$ to be non-negatively correlated on any rectangle in $\Re^{N+2}$, a plausible assumption in the case of oil and gas leases. Affiliation implies that the expected value of $V$, conditional upon $X$ and $R$, is non-decreasing in the realizations of $X$ and $R$. Affiliation also implies that the distribution of $R$, conditional upon $X$, satisfies the monotone likelihood ratio property with respect to the realizations of $X$.

Given the affiliation assumption, Hendricks, Porter, and Wilson have demon- 
strated that equilibrium imposes a set of restrictions upon the distribution of $B_{I}$, the bid of the informed potential buyer, and the distribution of $B_{U}$, the maximum bid submitted by the uninformed potential buyers. Let $G_{I}(b ; z)$ denote the distribution of $B_{I}$ conditional upon $Z=z, G_{U}(b ; z)$ denote the distribution of $B_{U}$ conditional upon $Z=z$, and $\bar{r}(z)$ denote the upper bounds of the support of $R$, conditional on $Z=z$. The relations between $G_{I}$ and $G_{U}$ for each realization $z$ of $Z$ are as follows:

$$
\begin{aligned}
& \text { (R1) } \frac{g_{U}(b ; z)}{G_{U}(b ; z)} \leq \frac{g_{I}(b ; z)}{G_{I}(b ; z)} \text { for } G_{I}(b ; z), G_{U}(b ; z)>0 ; \\
& \text { (R2) } G_{I}(b ; z)=G_{U}(b ; z) \text { for } b \geq \bar{r}(z) ; \\
& \text { (R3) } \exists \underline{b}(z) \ni G_{I}(\underline{b}(z) ; z)>G_{U}(0 ; z) \text { and } G_{I}(\underline{b}(z) ; z)=G_{U}(0 ; z)
\end{aligned}
$$

where for simplicity it is assumed that $G_{I}$ and $G_{U}$ are differentiable.

Relation (R1) states that the proportional rate of increase in the distribution of the maximum uninformed bid is never greater than the proportional rate of increase in the distribution of the informed bid. The inequality is strict in the support of the reserve price. Relation (R2) states that the the distributions are identical for bids above the support of the reserve price. Relation (R3) states that either the informed potential buyer submits his lowest bid $\underline{b}(z)$ with positive probability and $G_{I}$ is continuous, or there exists an interval around the lowest informed bid $\underline{b}(z)$ where the uninformed potential buyers never bid.

The above restrictions imply that uninformed potential buyers are less likely to participate than the informed potential buyer, but when they do participate they bid high rather than low. The probability of uninformed potential buyers participating and the range of bids at which they participate depends upon the distribution of the reserve price. In drainage auctions, the probability of rejection is quite high at bids below $\$ 1$ million. Consequently, the informed potential buyer is forced to bid close to his valuation when it lies in this range. This makes low bids unprofitable for uninformed potential buyers, since they would tend to win only when the value of the tract is less than the amount bid. By contrast, bids above $\$ 5$ million are rarely rejected, so uninformed potential buyers need to participate in this range to keep the informed potential buyers's profit margin at reasonable levels. Thus, roughly speaking, the implications of $(\mathrm{Rl})$ to $(\mathrm{R} 3)$ for the sample of drainage auctions are: first, a lower collective participation rate for non-neighbour firms; second, few non- 
neighbour bids below approximately $\$ 1$ million; and third, distributional equivalence at bids above $\$ 4$ or $\$ 5$ million.

Hendricks, Porter, and Wilson have used the empirical distributions of $B_{I}$ and $B_{U}$ to test these predictions and found remarkably strong support for the theory. The tests were based upon partitioning the set of all positive neighbour and highest nonneighbour bids into 8 equally sized subsets according to their rank, and comparing $\Delta \hat{G}_{I} / \hat{G}_{I}$ and $\Delta \hat{G}_{U} / \hat{G}_{U}$ for each subset, where the "^"s on the $G_{i} \mathrm{~s}(i=I, U)$ denote the empirical distribution function of the bids for the sample. Hendricks, Porter, and Wilson used non-parametric tests that apply the Wilcoxon rank sum statistic to establish statistical significance of one-sided departures from equivalence in the lower bid range, and equivalence in the upper bid range. Two reasons exist why the results of these tests need to be interpreted with caution.

First, the data are drawn from a truncated distribution: only tracts that received at least one bid are included. This creates a problem for testing the equivalence of $g_{I} / G_{I}$ and $g_{U} / G_{U}$ since the number of tracts on which no bids were submitted is needed to compute the denominators in these ratios. Fortunately, this is not a problem for testing an implication of (Rl) and (R2); viz., that $G_{I}$ stochastically dominates (in the first order sense) $G_{U}$. Letting $q$ denote the probability that a tract receives no bids the Hendricks, Porter, Wilson sample consists of draws from

$$
\frac{G_{I}(b)-q}{(1-q)} \text { and } \frac{G_{U}(b)-q}{(1-q)} \text {. }
$$

Since $0 \leq q \leq 1$, it follows that $G_{I}(b)$ equals $G_{U}(b)$ if and only if

$$
\frac{G_{I}(b)-q}{(1-q)}=\frac{G_{U}(b)-q}{(1-q)} .
$$

The second problem is more serious. Much of the variation in $Z$, the publicly available information variables, may be unobserved by the researcher. This implies that $B_{I}$ and $B_{U}$ are not necessarily independently distributed. Indeed Hendricks, Porter, and Wilson found this to be the case in the sample of drainage tracts. The lack of independence makes statistical inference difficult since the asymptotic distribution of test statistic is difficult to calculate. Almost surely, the distribution for the rank sum statistic is not a standard normal distribution. For this reason, the non-parametric tests are not definitive, and merely suggestive. 
Is it feasible to identify the probability law for $(V, X, R)$ and to test behavioural hypotheses structurally? From a theoretical perspective, the complicating feature of the model for drainage leases is the correlation between the random reserve price $R$ and the information of the informed potential buyer $X$. If the government had used a fixed reserve price (or, alternatively, if $R$ and $X$ are independent), then structural estimation would be feasible. The reason is that, regardless of the dimension of $X$, the information of the informed potential buyer can be summarized by his conditional expectation, $H=\mathrm{E}[V \mid X]$, which is a real-valued random variable. In that case, a bidding strategy of the informed potential buyer is simply a function which maps realizations of $H$ into bids. Letting $F$ denote the distribution function of $H$, the relationship between $F$ and the equilibrium bid distributions $G_{I}$ and $G_{U}$ when $R$ is fixed is given by

$$
\begin{aligned}
\sigma(h) & =\mathrm{E}[H \mid H \leq h] \\
G_{U}(b) & =F\left(\sigma^{-1}(b)\right) .
\end{aligned}
$$

See Hendricks, Porter, and Wilson (1992) for more details. One could conduct a parametric analysis similar in spirit to that outlined in the next sections, but from a practical perspective, the unobservability of important elements of $Z$ suggests that such an analysis is unlikely to succeed, at least for drainage lease data.

\section{Structural Models I: English Auctions}

Consider the following bidding environment. An indivisible object is to be sold at auction with an announced minimum (reserve) price $v_{0}$ set by the seller. There are $N$ potential buyers. The $i^{\text {th }}$ potential buyer has a valuation $v_{i}$ for the object, which is known to him, but not to his $(N-1)$ opponents. Heterogeneity across potential buyers in valuations is ascribed to independent draws from a common probability density function $f(v)$, with cumulative distribution function $F(v)$, having support upon the interval $[\underline{v}, \bar{v}]$. We shall assume that $v_{0}$ exceeds $\underline{v}$. The number of potential buyers $N$ and the distribution function $F(v)$ are assumed to be common knowledge. Potential buyers are assumed to be risk neutral with respect to winning the object.

This paradigm is known as the independent private values paradigm. It is the simplest possible bidding environment within which to test auction theory or to use auction theory to estimate the joint distribution function of the $N$ valuations $\left(v_{1}, v_{2}, \ldots, v_{N}\right)$. The reason is due to two important assumptions: the independence 
of valuations and the absence of any observable characteristics distinguishing buyers. Together, these two assumptions imply that the joint distribution function factors into $N$ identical marginal distributions. Thus, estimating $F$ is equivalent to recovering the entire joint distribution for the valuations of potential buyers. Furthermore, every bid (and, in particular, the winning bid) provides useful information concerning $F$. This is important since, in many instances, only winning bids are observed by the researcher. Throughout this section, we shall also assume that the researcher observes $N$.

The simplest auction from which to estimate $F$ is an English auction. Suppose the seller sets the reserve price at $v_{0}$, and then lets it rise more or less continuously as long as at least two potential buyers are willing to pay the announced price. Each potential buyer indicates his willingness to pay by some action which is observable not only to the seller but also to his opponents. Within this setting, the optimal strategy for each potential buyer is to participate as long as the announced sale price does not exceed his valuation. Thus, the winner is the potential buyer with the highest valuation. If he is the only potential buyer willing to participate at $v_{0}$, then the price he pays is $v_{0}$. Otherwise, he pays a price equal to the second highest valuation. (Here we ignore any discreteness in the bidding process.)

More formally, letting $v_{(i: N)}$ denote the $i^{\text {th }}$ highest order statistic for a sample of size $N$ from the distribution of $v$ and denoting the winning bid by $w$, the above bidding behaviour implies that $w$ equals $v_{(2: N)}$ whenever $w$ exceeds $v_{0}$. The probability density function for $w$ in this case is given by

$$
h_{E}(w)=N(N-1)[1-F(w)] F(w)^{N-2} f(w) .
$$

If $w$ equals $v_{0}$, then all that one can infer about $F$ is that $N-1$ draws are less than $v_{0}$ and one exceeds $v_{0}$. To calculate the probability of this event, introduce the indicator variable

$$
P_{i}= \begin{cases}1 & \text { if } v_{i} \geq v_{0} \\ 0 & \text { otherwise. }\end{cases}
$$

The number of participants at an auction is

$$
n=\sum_{i=1}^{N} P_{i}
$$


where $n$ is distributed binomially with parameters $N$ and $\operatorname{Pr}\left[P_{i}=1\right]=\left[1-F\left(v_{0}\right)\right]$. Thus, the probability of only one potential buyer participating, a winning bid of $v_{0}$, is

$$
h_{E}\left(v_{0}\right)=N F\left(v_{0}\right)^{N-1}\left[1-F\left(v_{0}\right)\right],
$$

while the probability of the object going unsold, $n=0$ or $w=0$, is

$$
h_{E}(0)=N F\left(v_{0}\right)^{N} .
$$

Notice that the density function for the winning bid has discrete mass points as well as a continuous density even though the density of $v$ is strictly continous.

Since the reserve price $v_{0}$ exceed $\underline{v}$, the number of actual bidders (participants) at an auction $n$ is endogenous, and typically less than the number of potential buyers $N$. Only those potential buyers with valuations exceeding the reserve price $v_{0}$ participate.

In general, auctions are not identical, and can differ according to certain characteristics. Letting $t$ index a random sample of $T$ auctions, we adopt the parametric formulation

$$
F_{t}(v)=F\left(v ; \theta, Z_{t}\right)
$$

where $Z_{t}$ is a vector of characteristics that are observable not only to all of the potential buyers, but also to the researcher. Thus, $F$ represents the distribution of the unobserved heterogeneity embodied in private valuations conditional upon the characteristics of the object being sold. With independence across auctions as well as variation in the number of potential buyers $N_{t}$, the likelihood function takes the form

$$
L(\theta)=\prod_{t=1}^{T} \frac{h_{E}\left(v_{0} ; \theta, Z_{t}, N_{t}\right)^{D_{t}} h_{E}\left(w_{t} ; \theta, Z_{t}, N_{t}\right)^{1-D_{t}}}{\left[1-h_{E}\left(0 ; \theta, Z_{t}, N_{t}\right)\right]}
$$

where

$$
D_{t}= \begin{cases}1 & \text { if } n_{t}=1 \\ 0 & \text { otherwise. }\end{cases}
$$

Here the indicator variable $D_{t}$ is a switch which describes the level of competition. The contributions for one participant and for more than one participant are scaled by one minus the contribution for no participants to reflect the fact that most samples are truncated samples: data concerning auctions for which no one attends are usually unavailable. An estimate of the unknown value of $\theta$ can be calculated by maximizing $L(\theta)$ or $\log L(\theta)$ with respect to $\theta$. 
The behavioural hypothesis of the model is that potential buyers bid independently and losers tell the truth. Each potential buyer drops out of the bidding when the price reaches his valuation, thereby revealing his valuation. The optimality of this strategy does not depend upon the strategies chosen by opponents. Hence, the equilibrium is a dominant strategy. Unfortunately, the absence of strategic play makes it difficult to test the theory, assuming $F$ is unknown. Any assumption concerning $F$ is an assumption concerning the distribution of bids (or the winning bid) since the bid function is essentially the identity function.

Paarsch $(1989,1991)$ has applied this model to data concerning timber sales in the province of British Columbia, Canada. In British Columbia, the Minister of Forests sets aside a portion of each year's allowable cut to sell to eligible loggers and sawmillers through a series of public auctions held under the Small Business Forest Enterprise Program (SBFEP). To be eligible, a logger or mill-owner must be registered (at a cost of $\$ 100$ per year) and must not hold more than two SBFEP contracts at the time of the sale. Paarsch considered only Type 1 participants in the SBFEP, those who were loggers, since only a few auctions for sawmillers (Type 2 auctions) were held in any district. Paarsch used the number of eligible Type 1 SBFEP registrants in a Forest District as a measure of $N$ for auctions held in that district, since registrants rarely participated at auctions outside of their district. The number of eligible Type 1 SBFEP registrants in a Forest District is available to the public at the district office, and varies across sales.

The object being sold is the right to harvest a stand of timber. Prior to the auction, the Ministry of Forest makes available a report (called the "timber cruise") which provides information concerning the terrain and the accessibility of the stand as well as the quality and volume of timber on the stand. In addition, potential buyers can and do inspect the stand themselves. The Ministry of Forests assigns a minimum (reserve) price (known as the "upset" price) per cubic metre, $v_{0}^{k}$, for each species $k$ harvested. A bid is then a single number which the buyer agrees to pay for each cubic metre of timber harvested in addition to the minimum price. Thus, if awarded the cutting rights at price $s$, the buyer pays the Ministry of Forests $s+v_{0}^{k}$ for each cubic metre of species $k$ harvested. These prices are known as "stumpage rates".

Paarsch assumed that each potential buyer's valuation of a stand is proportional to the volume of standing timber (or some weighted average of the volumes of the 
different species) less a fixed cost. The proportionality factor is the sum of two observable components, prices for logs and costs of transport, and an unobservable component which Paarsch interprets as harvesting costs. Prices for logs and costs of transport as well as timber volumes, and the number of potential buyers are the sources of observed heterogeneity. Their variation across auctions is used to identify the parameters of the net return function. Harvest costs are assumed to be independently and identically distributed across potential buyers and timber sales. Paarsch considered two specifications for the probability law, the Pareto and the Weibull. Estimates of their parameters, together with those of the return function, are obtained using the method of maximum likelihood as described above. From Paarsch's estimates, one can calculate the amount of rent garnered by the government

$$
\mathrm{E}\left[v_{(2: N)}\right]
$$

which was about $\$ 16,500$ for the average sale in the sample.

\section{Structural Models II: First-Price Sealed-Bid Auctions}

Consider once again the independent private values paradigm, but where the sale mechanism is a first-price sealed-bid auction. At these auctions, each potential buyer is asked to submit his bid privately to the seller. The object is then sold to the highest bidder at a price equal to his bid, provided this bid is at least as high as the reserve price. If more than one potential buyer makes the same high bid, then the object is allocated according to some rationing rule which is independent of the reserve price. In this setting, a (pure) strategy for the $i^{\text {th }}$ potential buyer is a real valued function $\hat{\sigma}_{i}$ of the realization for $V_{i}$. Given a strategy profile $\hat{\sigma}=\left(\hat{\sigma}_{1}, \hat{\sigma}_{2}, \ldots, \hat{\sigma}_{N}\right)$, the expected pay-off to the $i^{\text {th }}$ potential buyer with valuation $v_{i}$ from bidding $s_{i}=\hat{\sigma}_{i}\left(v_{i}\right)$ is

$$
\left(v_{i}-s_{i}\right) \prod_{j \neq i} F\left(\hat{\sigma}_{j}^{-1}\left(s_{i}\right)\right)
$$

where $\hat{\sigma}_{j}^{-1}$ denotes the inverse (possibly a set-value correspondence) of potential buyer $j$ 's strategy. The first term in parentheses of (4.1) represents potential buyer $i$ 's profit margin, while the second term of (4.1) represents the probability of potential buyer $i$ 's winning the auction given bid $s_{i}$. Note that the latter depends upon the strategies of his opponents and the distribution function of their valuations. Consequently, 
potential buyer $i$ 's optimal strategy depends upon his beliefs concerning the bidding rules of his opponents as well as the distribution of their valuations.

To solve the game, we use a solution concept known as Bayesian-Nash equilibrium. This concept requires each potential buyer's conjectures concerning the strategies played by his opponents to be correct, and that his strategy is a best response to these conjectures. More precisely, a Bayesian-Nash equilibrium is a strategy profile $\sigma$ such that for each $i=1, \ldots, N, \sigma_{i}$ is a best response to $\sigma_{-i}=$ $\left(\sigma_{1}, \ldots, \sigma_{i-1}, \sigma_{i+1}, \ldots, \sigma_{N}\right)$ for every realization of $V_{i}$. In what follows, we shall focus upon equilibria at which all potential buyers use the same strategy, and that strategy is a strictly increasing, differentiable function of $V$.

The first-order condition for optimality of $s_{i}$ is

$$
\left(v_{i}-s_{i}\right)\left[\sum_{j \neq i} f\left(\hat{\sigma}_{j}^{-1}\left(s_{i}\right)\right) \frac{d \hat{\sigma}_{j}^{-1}\left(s_{i}\right)}{d s_{i}} \prod_{k \neq j} F\left(\hat{\sigma}_{k}^{-1}\left(s_{i}\right)\right)\right]-\prod_{j \neq i} F\left(\hat{\sigma}_{j}^{-1}\left(s_{i}\right)\right)=0 .
$$

If (4.2) is assumed to hold for all values of $V_{i}$ and the condition of symmetry is imposed, then (4.2) becomes a differential equation in $\hat{\sigma}$

$$
\hat{\sigma}^{\prime}(v)+\frac{(N-1) f(v)}{F(v)} \hat{\sigma}(v)=\frac{(N-1) v f(v)}{F(v)}
$$

where $\hat{\sigma}^{\prime}(v)$ is the derivative of $\hat{\sigma}(v)$ with respect to $v$. Using the boundary condition $\hat{\sigma}\left(v_{0}\right)=v_{0}$, this differential equation can be solve for valuations above the reserve price to obtain the equilibrium bid function

$$
\sigma(v)=v-\frac{\int_{v_{0}}^{v} F(u)^{N-1} d u}{F(v)^{N-1}} \quad v \geq v_{0} .
$$

Equation (4.4) states that potential buyers with valuations above the reserve price bid their valuation less a "shading" factor, the magnitude of which reflects the amount of competition $N$, where a potential buyer is in the distribution $F(v)$, and the reserve price $v_{0}$. Note that $\sigma$ is strictly increasing in $v$, as required by the initial hypothesis. For those with valuations less than $v_{0}$, no bid (which we denote by zero) is submitted. This participation condition is identical to that at English auctions. It implies that the number of participants at the auction $n$ is once again endogenous.

The winner of the auction will be the potential buyer with the highest valuation $v_{(1: N)}$. The winning bid function is monotonic in $v_{(1: N)}$, and its distribution is related 
to that of the largest order statistic for a sample of size $N$ from the distribution of $v$. This distribution is different from that for the winning bid at an English auction, although the revenue equivalence proposition implies that the expected value of the winning bid at a first-price sealed-bid auction equals that at an English auction; see Riley and Samuelson (1991).

Exploiting the fact that (4.4) is a monotonic function of $v$ (the higher is a potential buyer's valuation, the more he will bid) provides one strategy for interpreting field data; see, for example, Paarsch $(1989,1991,1992)$. Because the bidding rules are functions of the random variable $v$, the bids are also random variables and their densities are related to $f(v)$. Thus,

$$
G_{S}(s)=F\left(\sigma^{-1}(s)\right) \text { and } g_{S}(s)=\frac{d G_{S}(s)}{d s}=\frac{f\left(\sigma^{-1}(s)\right)}{\sigma^{\prime}\left(\sigma^{-1}(s)\right)}
$$

Note that the support of the bid distribution function is on the interval $\left[v_{0}, \bar{s}\right]$ where $\bar{s}=\sigma(\bar{v})$.

An interesting question to ask is whether another latent distribution exists (other than $F$ ) which would be consistent with the observed data $G_{S}$. This is a nontrivial identification problem in the case of first-price sealed-bid auctions because, eventhough $\sigma$ is invertible in $v$, it depends upon $F$.

For the case of first-price sealed-bid auctions within the independent private values paradigm, Donald and Paarsch (1992) have elaborated upon a set of necessary and sufficient conditions which permit identification by working directly off of (4.4). These turn out to be quite innocuous, and to be met by virtually all parametric distributions which a researcher might consider using in practice.

Perrigne, Simioni, and Vuong (in progress) (as discussed in Elyakime, Laffont, Loisel, and Vuong [forthcoming]) work directly with the differential equation (4.3). They substitute the relations given in (4.5) into the differential equation (4.3), and solve for $v$

$$
v=s+\left[\frac{(N-1) g_{S}(s)}{G_{S}(s)}\right]^{-1} \equiv \xi(s) .
$$

Perrigne, Simioni, and Vuong show that the necessary and sufficient conditions for identification are that $\xi(s)$ is strictly increasing in $s$, and that $\lim _{s \rightarrow v_{0}} \xi(s)=v_{0}$. In words, if $g_{S}(s) / G_{S}(s)$ is well behaved, then there exists a unique $F$ that makes $G_{S}$ the distribution for any one of the equilibrium bids. Note that $\xi(s)$ is simply the 
inverse function $\sigma^{-1}(v)$, and that this approach to identification does not require an explicit solution to (4.3).

\subsection{Maximum Likelihood}

To keep the analysis similar to that of English auctions, we shall assume that the researcher uses only the winning bid. ${ }^{2}$ The winning bid is a simple function of the $\left\{v_{i}\right\}_{i=1}^{N}$. Hence, its density is related to $f(v)$. The density of the winning bid at a first-price sealed-bid auction $w=\sigma\left(v_{(1: N)}\right)$, denoted $h_{S}(w)$, is

$$
\begin{aligned}
h_{S}(w) & =\frac{N F\left(\sigma^{-1}(w)\right)^{N-1} f\left(\sigma^{-1}(w)\right)}{\sigma^{\prime}\left(\sigma^{-1}(w)\right)} \\
& =\frac{N F\left(\sigma^{-1}(w)\right)^{N}}{(N-1)\left(\sigma^{-1}(w)-w\right)} .
\end{aligned}
$$

The last equality in (4.7) is obtained by substituting in the derivative of $\sigma$. When the object goes unsold, one only knows that $v_{(1: N)}$ is less than $v_{0}$, which one can calculate using the method presented in section 3 . Hence,

$$
\operatorname{Pr}[n=0]=F\left(v_{0}\right)^{N}
$$

Maximum likelihood estimation requires that the researcher adopt a parametric specification for the probability law for each auction $t=1, \ldots, T$. As in section 3 , let

$$
F_{t}(v)=F\left(v ; \theta, Z_{t}\right)
$$

where $Z_{t}$ is a vector of observed covariates. Estimation by the method of maximum likelihood is not as straightforward as in the case of English auctions. The difficulty arises from the fact that the equilibrium bid function at first-price sealed-bid auctions depends upon $F\left(v ; \theta, Z_{t}\right)$. This in turn implies that $\bar{s}\left(\theta, Z_{t}, N_{t}\right)$, the upper bound of support of $h_{S}\left(w ; \theta, Z_{t}, N_{t}\right)$, depends upon the parameters of interest, which violates the standard regularity conditions required to demonstrate the consistency of the maximum likelihood estimator and to derive its asymptotic distribution.

2 In most instances, every bid above the reserve price is observed, but the modifications to the analysis are straightforward. 
Donald and Paarsch (1991) have proposed a maximum likelihood estimator. The estimator requires maximizing the likelihood of the observed sample subject to the constraints that the bidding outcomes be consistent with the implied upper bounds of the bid distributions. More formally, the maximum likelihood estimator is defined as the solution to the following non-linear programming problem:

$$
\max _{<\theta>} \sum_{t=1}^{T}\left[\log h_{S}\left(w_{t} ; \theta, Z_{t}, N_{t}\right)-\log \left[1-F\left(v_{0 t} ; \theta, Z_{t}\right)^{N_{t}}\right]\right]
$$

subject to

$$
\begin{aligned}
w_{1} & \leq \bar{s}\left(\theta, Z_{1}, N_{1}\right) \\
w_{2} & \leq \bar{s}\left(\theta, Z_{2}, N_{2}\right) \\
\vdots & \\
w_{T} & \leq \bar{s}\left(\theta, Z_{T}, N_{T}\right) .
\end{aligned}
$$

The second term within the large brackets of the sum defining the objective function represents a correction for the fact that auction samples are typically truncated samples: only those auctions at which at least one potential buyer competes enter data sets.

Donald and Paarsch have demonstrated that the maximum likelihood estimator is not simply consistent, but super-consistent: the estimator converges to the true parameter vector at rate $T$ instead of the typical rate $\sqrt{T}$, a fact arising from the use of extreme value statistics instead of averages to define the estimator. This quick rate of convergence is very important in applications to auctions where sample sizes of 50 are common, and those of 200 would be considered large. The solution which defines the maximum likelihood estimator typically obtains at the intersection of the constraints, so the properties of the perturbed optimum of the logarithm of the likelihood function are determined solely by the constraints. Consequently, the distribution theory for the estimator is quite complicated. Indeed, with discrete covariates, Donald and Paarsch show that the limiting distributions of the estimator falls within the exponential family of limiting distributions.

Paarsch $(1989,1991)$ has applied the above structural framework to data concerning timber sales in British Columbia, Canada. Several Forest Districts used first-price sealed-bid auctions rather than English auctions to sell the right to harvest 
timber. Moreover, the choice of mechanism appears random - uncorrelated with economic factors such as prices for logs, volumes of standing timber, or "upset rates" - suggesting that the underlying probability law for the valuations of potential buyers may have been the same across the two types of auctions. If so, the estimate of $F$ obtained from the two data sets should be the same. The results did not support this hypothesis. In particular, the participation behaviour of potential buyers seemed to differ accross the two mechanisms, since the fraction of auctions at which the winning bid was the reserve (upset) price was significantly higher at English auctions than at first-price sealed-bid auctions. Paarsch also found some evidence suggesting that potential buyers were not risk neutral with respect to winning the auction. In any case, from Paarsch's estimates for the risk neutral specification, one can calculate the amount of rent garnered by the government; it was negative for the average covariates of the sample.

This empirical work illustrates the potential of structural models. Theory predicts that average revenues at English and first-price sealed-bid auctions should be the same if valuations are independently and identically distributed and potential buyers bid competitively. In the case of timber sales in British Columbia, this implication does not appear to hold, even after one accounts for observable heterogeneity. The reasons for this may be mis-specification of the structural elements or inappropriate behaviour. In either case, structural methods are needed to evaluate the significance of differences in outcomes, and to explain them.

\subsection{Simulated Non-Linear Least Squares}

The main drawback to applying the method of maximum likelihood is numerical complexity. To calculate the contribution of $\left(w_{t}, Z_{t}, N_{t}\right)$ to the logarithm of the likelihood function, one needs to compute the function $\sigma^{-1}\left(w_{t} ; \theta, Z_{t}, N_{t}\right)$. But except in special cases, this function (and, for that matter, $\bar{s}\left(\theta, Z_{t}, N_{t}\right)$ ) is non-analytic, and will typically need to be evaluated numerically at every observation and every trial value of $\theta$. The computational burden of this exercise can be high, and can effectively limit the class of distributions which the researcher can entertain.

Laffont, Ossard, and Vuong (1991) have proposed an alternative estimation strategy which can circumvent some of these computational difficulties in certain circumstances. The strategy of Laffont, Ossard, and Vuong is called the method 
of "simulated non-linear least squares" and it is closely related to the method of "simulated moments" proposed by McFadden (1989) and Pakes and Pollard (1989).

Laffont, Ossard, and Vuong (1991) have noted that within the independent private values paradigm, the equilibrium bid function for the $t^{\text {th }}$ auction can be written as the following conditional expectation:

$$
\sigma_{t}(v)=\mathrm{E}\left[\max \left\{v_{0 t}, v_{\left(2: N_{t}\right)}\right\} \mid v_{\left(1: N_{t}\right)}=v\right]
$$

Since the expectation of the winning bid at auction $t$ corresponds to the expectation of second highest private value, ${ }^{3}$ this implies that

$$
\mathrm{E}\left[w_{t}\right]=\mu\left(\theta, Z_{t}, N_{t}\right)=\mathrm{E}\left[\max \left\{v_{0 t}, v_{\left(2: N_{t}\right)}\right\}\right]
$$

Integrating over all possible realizations of the second highest valuation then yields

$$
\begin{aligned}
& \mu\left(\theta, Z_{t}, N_{t}\right)= \\
& \int_{0}^{\infty} \ldots \int_{0}^{\infty} \max \left\{v_{0 t}, v_{\left(2: N_{t}\right)}\right\} f\left(v_{1} ; \theta, Z_{t}, N_{t}\right) \ldots f\left(v_{N_{t}} ; \theta, Z_{t}, N_{t}\right) d v_{1} \ldots d v_{N_{t}} .
\end{aligned}
$$

Equation (4.8) is used as the basis of the proposed simulated non-linear least squares estimator.

The idea is straigthforward. For each of $t=1, \ldots, T$ observations, $J$ independent simulation samples of size $N_{t}$ are drawn; (4.8) is then approximated by the sample mean of this function for each of the simulated samples. Following Gourieroux and Monfort (1990), Laffont, Ossard, and Vuong use the concept of the "importance function" (common in Bayesian Monte Carlo integration) to get around the problem that the parameter vector to be estimated $\theta$ is part of the function to be simulated $\mu(\theta, Z, N)$. Letting $\psi_{t}(\cdot)$ denote the importance function - which the researcher must specify ex ante - Laffont, Ossard, and Vuong estimate (4.8) by

$$
\bar{m}\left(\theta, Z_{t}, N_{t}\right)=\frac{1}{J} \sum_{j=1}^{J} m_{j}\left(\theta, Z_{t}, N_{t}\right)
$$

3 When potential buyers are risk averse, this equality will be violated. Also, within other paradigms this equality will not, in general, hold. 
where

$$
m_{j}\left(\theta, Z_{t}, N_{t}\right)=\max \left\{v_{0 t}, v_{\left(2: N_{t}\right) t}^{j}\right\} \frac{f_{t}\left(v_{1 t}^{j} ; \theta\right) \ldots f_{t}\left(v_{N_{t} t}^{j} ; \theta\right)}{\psi_{t}\left(v_{1 t}^{j}\right) \ldots \psi_{t}\left(v_{N_{t} t}^{j}\right)}
$$

Here, each of the $v_{i t}^{j}$ s represents an independent draw for a random variable having the probability density function $\psi_{t}(\cdot), i=1, \ldots, N_{t} ; j=1, \ldots, J$; and $t=1, \ldots, T$.

Laffont, Ossard, and Vuong have noted that using $\bar{m}\left(\theta, Z_{t}, N_{t}\right)$ as $\mu\left(\theta, Z_{t}, N_{t}\right)$ when minimizing the objective function

$$
\sum_{t=1}^{T}\left(w_{t}-\bar{m}\left(\theta, Z_{t}, N_{t}\right)\right)^{2}
$$

with respect to $\theta$ will produce an inconsistent estimator of $\theta$ for any finite number of simulations $J$ because $\bar{m}\left(\theta, Z_{t}, N_{t}\right)$ estimates $\mu\left(\theta, Z_{t}, N_{t}\right)$ with error. They have derived the simulated non-linear least squares estimator of $\theta$, which is consistent for a finite fixed $J$ simulations, by minimizing the following objective function:

$$
Q(\theta)=\sum_{t=1}^{T}\left[\left(w_{t}-\bar{m}\left(\theta, Z_{t}, N_{t}\right)\right)^{2}-\frac{1}{J(J-1)} \sum_{j=1}^{J}\left(m_{j}\left(\theta, Z_{t}, N_{t}\right)-\bar{m}\left(\theta, Z_{t}, N_{t}\right)\right)^{2}\right]
$$

with respect to $\theta$. The bias introduced by the pre-estimation error in $\bar{m}\left(\theta, Z_{t}, N_{t}\right)$ is eliminated asymptotically by introducing the second term of $Q(\theta)$, which represents an estimate of the sample variance of $\bar{m}\left(\theta, Z_{t}, N_{t}\right)$. Laffont, Ossard, and Vuong have shown that the simulated non-linear least squares estimator is distributed asymptotically normal, and they derive an estimator of the variance-covariance matrix for the simulated non-linear least squares estimator.

Laffont, Ossard, and Vuong have applied their method of estimation to data from daily sales of eggplants at a market in Marmande, France during the summer of 1990. Each day several lots of eggplants of variable size - 15 to 350 kilograms - are sold sequentially using a descending auction. The seller announces a reserve price per unit (i.e., a price per kilogram) and then starts the bidding at a very high unit price. The price drops quickly until one of the potential buyers makes a bid. The first potential buyer who makes a bid before the reserve price is reached wins the lot and pays his bid. This one-shot descending (or Dutch) auction is strategically equivalent to a first-price sealed-bid auction when the valuations of potential buyers 
are independently and identically distributed. Laffont, Ossard, and Vuong assumed that $F$ belongs to the log-normal family of distributions.

Laffont, Ossard, and Vuong faced two difficulties in their application which are likely to present problems for any researcher seeking to do empirical work on this type of auction. At Dutch auctions, only the winning bid is observed since losers are not required to submit their bids. This makes it difficult for a researcher to measure the number of potential buyers. Laffont, Ossard, and Vuong inferred the presence of at least eleven buyers, since there were eleven different winners in their sample. But this does not mean that each potential buyer attended every auction, nor does it account for the possibility of potential buyers who do not win. The authors resolved this problem by treating $N$ as a parameter to be determined by the data.

The second problem is to justify the independent private values paradigm. The potential buyers are agents of retail sellers who serve distinct markets, and place orders with their agents at specific prices before the opening of the market each day. The sources of observable heterogeneity common to all potential buyers are quantity and quality of supply, and variables measuring the general state of demand. Variation in valuations across retailers is then attributed to idiosyncratic factors in each retailer's daily market. Furthermore, for each retailer, these factors are random variables which are assumed to be independently and identically distributed across market days. Thus, the sample selected consisted of one auction per day for each market day in the period from June to October of 1990. Nevertheless, the assumption of independence across market days seems quite strong.

\subsection{Non-Parametric Estimation}

One criticism of the method of maximum likelihood or the method simulated nonlinear least squares is that an explicit distributional assumption must be made concerning $f(v)$. Recently, Elyakime, Laffont, Loisel, and Vuong (forthcoming) have developed a non-parametric approach to estimating $F$ at first-price sealed-bid auctions. They exploit equation (4.6), which expresses the unobserved valuation of a potential buyer in terms of his bid and the potentially estimable quantity $g_{S}(s) / G_{S}(s)$. Hence, in contrast to parametric methods, this approach requires knowledge of all of the bids, not just the winning bid. The main behavioural assumptions are that each potential buyer is bidding optimally against the bidding distribution generated by his 
opponent's strategies, that all potential buyers are using the same strategy, and that the strategy is increasing in $v$.

Application of the procedure consists of two steps. The sample is the set of all pairs $\left\{\left(s_{\ell}, Z_{\ell}\right): \quad \ell=1, \ldots, \sum_{t=1}^{T} n_{t}\right\}$, where $\sum_{t=1}^{T} n_{t}$ is the total number of bids submitted at the $T$ auctions. Treating each observation as an independent draw, the first step consists of estimating the conditional density $g_{S}(s \mid Z)$ non-parametrically. This step produces $\hat{g}_{S}(s \mid Z)$ and $\hat{G}_{S}(s \mid Z)$ which can be used in the second step to estimate the "hazard rate"

$$
\frac{g_{S}(s \mid Z)}{G_{S}(s \mid Z)}
$$

The second step consists of plugging in the non-parametric estimates of the "hazard rate" into (4.6) and evaluating $\xi(s \mid Z)$ at each bid $s_{\ell}$ to generate a fitted sample $\left\{\left(s_{\ell}, Z_{\ell}\right): \ell=1, \ldots, \sum_{t=1}^{T} n_{t}\right\}$. This sample can be used to obtain a non-parametric estimate of the bidding strategy $\hat{\xi}^{-1}(s \mid Z)$ and of the conditional density of valuations $\hat{f}(s \mid Z)$.

This non-parametric estimation method identifies the underlying probability law for the valuations of buyers. Moreover, as Elyakime, Laffont, Loisel, and Vuong have pointed out, it also provides a test of the behavioural hypothesis. The non-parametric estimates of $g_{S}(s) / G_{S}(s)$ can produce an estimate of $\hat{\xi}(s \mid Z)$ which is not everywhere increasing. Nor does the procedure require $\hat{\xi}\left(v_{0} \mid Z\right)$ to equal $v_{0}$. These properties are necessary implications of the theory. Consequently, if the estimated bid strategy is increasing, and passes through the reserve price, this is evidence consistent with the theory.

Elyakime, Laffont, Loisel, and Vuong have applied this method to data concerning timber sales in Tarn, France. A coöperative of timber owners sells the right to harvest standing timber to sawmills. The market is held twice each year. The characteristics of each lot are described in a booklet which is freely available to potential buyers prior to each sale. In addition, potential buyers can inspect the stands. Each stand is auctioned successively the day of the sale at first-price sealed-bid auction. The main source of observable heterogeneity which Elyakime, Laffont, Loisel, and Vuong have considered is the number of actual bidders and the percentage of saw timber.

The interesting theoretical feature of these auctions is that the seller's reservation price is not announced until after the bids have been submitted. Hence, any interested 
buyer must submit his bid in ignorance of the reserve price. This is particularly useful from an empirical perspective since in the absence of participation costs it implies no truncation of the bids. However, it does introduce an asymmetry into the model. From the perspective of potential buyers, the seller is another potential buyer, but one who has a different pay-off function. This implies a different bidding strategy for the seller from those of the potential buyers. Consequently, the optimal strategy of potential buyers cannot be obtained explicitly, and the researcher must work with a differential equation like (4.3) as the basis of the empirical model.

\subsection{Remarks}

Parametric methods require the researcher to assume that $F$ belongs to a particular family of distributions. Hence, even if one takes as given that valuations of potential buyers are independently and identically distributed random variables, the hypothesis being tested is always a joint hypothesis involving both functional form and behavioural assumptions. This is not uncommon in economics. Consider, for example, much of the research devoted to testing consumer and producer theory.

The maximum likelihood estimator is feasible in bidding environments where the differential equation(s) characterizing equilibrium behaviour can be solved explicitly, and the solution has a closed-form. The simulated non-linear least squares estimator requires an explicit solution to the differential equation, but does not impose any further restrictions. Such solutions generally exist when potential buyers are risk neutral and valuations are independently and identically distributed random variables.

Paarsch (forthcoming) has compared the performance of the maximum likelihood and simulated non-linear least squares estimators. The fact which emerges from his research is that the simulated non-linear least squares estimator only finds a neighbourhood of the true parameter value some of the time when the number of simulations or the number of observations (or both) are small.

Although the maximum likelihood estimator requires imposing much more structure upon $f(v)$ than the simulated non-linear least squares estimator, it has one redeeming feature: the maximum likelihood estimator coverges at rate $T$, while the simulated non-linear least squares estimator only converges at rate $\sqrt{T}$. In practical terms, this means that an empirical researcher can obtain as much accuracy with a sample size of 50 when using the method of maximum likelihood as he could with a 
sample size of 2500 when using the method of simulated non-linear least squares. This is of particular relevance when investigating field data from actual auctions because sample sizes of 50 are common, and those of 200 would be considered large.

The non-parametric estimator is computationally quite simple. It requires no prior restrictions on the underlying probability law for the valuations, except for independence. It provides a weak test of the theory in that $\hat{\xi}(s)$ need not be strictly increasing and $\hat{\xi}\left(v_{0}\right)$ need not equal $v_{0}$. The main disadvantage of the method is inefficiency: large sample size are required, particularly if there are several important covariates.

\section{Summary and Conclusions}

Identifying the joint distribution of valuations for potential buyers and testing the theory is a formidable task, even in the relatively simple independent private values paradigm. The non-structural analysis of Hendricks, Porter, and Wilson (1992) on drainage lease auctions has provided considerable support for the notion that gametheoretic models of bidding provide a good description of how potential buyers behave at auctions. The evidence from the structural analyses of Paarsch $(1989,1991,1992)$ as well as Laffont, Ossard, and Vuong (1991) has also been encouraging but less conclusive, partly due to data restrictions.

The principal data restriction concerns the endogeneity of the number of participants when the seller sets a reserve price. In such cases, the number of actual bidders $n$ is not a valid measure of the level of competition. Instead, it must be viewed as a random variable whose distribution is determined by the same factors which determine the distribution of bids: the number of potential buyers, the reserve price, the joint distribution of valuations, and (possibly) the equilibrium bid strategies. ${ }^{4}$ The econometric model should be extended to explain the number of bids as well as the bids. The difficulty in doing so, however, is that the researcher typically does not have accurate measures of the number of potential buyers $N$. If $N$ is unknown to buyers, then a model of stochastic entry similar to that of McAfee and Vincent (1992) is required.

4 In the independent private values paradigm, the participation decision does not depend upon the bidding strategies of rivals since each potential buyer participates if and only if his valuation exceeds the reserve price. This is not the case when valuations are affiliated. 
The main challenge, however, is to develop tractable empirical models of bidding environments where the valuations of potential buyers are neither independently nor identically distributed. The theory of bidding in the general affiliated values model, which nests both the independent private values and the common value models, has been worked out by Milgrom and Weber (1982a). The econometric implementation of this model is still in its infancy but, if successful, would enhance significantly the domain of application. (See Laffont and Vuong [1992] for a discussion of some of the problems that are involved, particularly that of identification.) Tests of the independent private values paradigm would also be useful, especially for evaluating the structural models discussed above. Donald and Paarsch (1993b) have developed specification tests which use information from both the parametric and non-parametric approaches to test distributional assumptions and to examine for departures from independence, but more work must be done in this area.

On a more speculative note, the approach outlined by Elyakime, Laffont, Loisel, and Vuong (forthcoming) seems quite promising, at least for the independent private values paradigm. The basic idea behind their approach is to use the first-order condition for potential buyer $i$ to express his valuation $v_{i}$ in terms of his bid $s_{i}$ and the sum of the empirical "hazard rates" of his rivals' bid distributions evaluated at $s_{i}$. The researcher can then use this equation together with the sample of bids for potential buyer $i$, to estimate the probability law for his valuation. The assumption here is that potential buyer $i$ is bidding optimally against the observed bidding behaviour of his rivals. Note that there is no need to assume that potential buyers have the same probability law. It may then be possible to test for equilibrium behaviour by determining whether, given the estimated probability density function for potential buy $i$ 's valuation, optimization by potential buyer $i$ 's rivals generates bid distributions which are consistent with the observed bid distributions. 


\section{B. Bibliography}

Cox, J., B. Roberson, and V. Smith (1982) 'Theory and Behavior of Single Object Auctions.' In Research in Experimental Economics, Volume 2, edited by V. Smith. (Greenwich, CT: JAI Press)

Cox, J., V. Smith, and J. Walker (1985) 'Expected Revenue in Discriminative and Uniform Price Sealed-Bid Auctions.' In Research in Experimental Economics, Volume 3 , edited by V. Smith. (Greenwich, CT: JAI Press)

Cox, J., V. Smith, and J. Walker (1988) 'Theory and Individual Behavior of FirstPrice Auctions.' Journal of Risk and Uncertainty, 1, 61-99

Donald, S. and H. Paarsch (1991) 'Maximum Likelihood Estimation in Empirical Models of Auctions.' Typescript, Department of Economics, University of British Columbia

Donald, S. and H. Paarsch (1992) 'Identification in Empirical Models of Auctions.' Research Report 9216, Department of Economics, University of Western Ontario

Donald, S. and H. Paarsch (1993a) 'Piecewise Maximum Likelihood Estimation in Empirical Models of Auctions.' International Economic Review, 34, 121-148

Donald, S. and H. Paarsch (1993b) 'Tests of Mis-specification for Empirical Models of Auctions within the Independent Private Values Paradigm.' Typescript, Department of Economics, University of Western Ontario

Dyer, D., J. Kagel, and D. Levin (1989) 'A Comparison of Naive and Experienced Bidders in Common Value Offer Auctions: A Laboratory Analysis.' Economic Journal, 99, 108-115

Elyakime, B., J.-J. Laffont, P. Loisel, and Q. Vuong (forthcoming) 'First-Price Sealed-Bid Auctions with Secret Reservation Prices.' Annales d'Economie et de Statistique

Engelbrecht-Wiggans, R., P. Milgrom, and R. Weber (1983) 'Competitive Bidding and Proprietary Information.' Journal of Mathematical Economics, 11, 161-169

Gourieroux, C. and A. Monfort (1990) 'Simulation Based Inference in Models with Heterogeneity.' Annales d'Économie et de Statistique, 20, 69-107

Hendricks, K. (1993) 'Bidding Behavior in OCS Drainage Auctions: Theory and Evidence.' European Economic Review, 37, xx-xx

Hendricks, K. and R. Porter (1988) 'An Empirical Study of an Auction with Asymmetric Information.' American Economic Review, 78, 865-883 
Hendricks, K. and R. Porter (1992) 'Joint Bidding in Federal OCS Auctions.' American Economic Review, 82, 506-511

Hendricks, K., R. Porter, B. Boudreau (1987) 'Information, Returns and Bidding Behavior in OCS Auctions: 1954-1969.' Journal of Industrial Economics, 35, $517-542$

Hendricks, K., R. Porter, and R. Spady (1989) 'Random Reservation Prices and Behavior in OCS Drainage Auctions.' Journal of Law and Economics, 32, S83S106

Hendricks, K., R. Porter, and C. Wilson (1992) 'Auctions for Oil and Gas Leases with an Informed Bidder and a Random Reservation Price." Typescript, Department of Economics, University of British Columbia

Kagel, J. and D. Levin (1986) 'The Winner's Curse and Public Information in Common Value Auction.' American Economic Review, 76, 894-920.

Laffont, J.-J., H. Ossard, and Q. Vuong (1991) 'Econometrics of First-Price Auctions." Document de Travail No. 7, Institut d'Economie Industrielle, Université des Sciences Sociales de Toulouse

Laffont, J.-J. and Q. Vuong (1993) 'Structural Analysis of Descending Auctions.' European Economic Review, 37, xx-xx

McAfee, R. and J. McMillan (1987) 'Auctions and Bidding.' Journal of Economic Literature, 25, 699-738

McAfee, R. and D. Vincent (1992) 'Updating the Reserve Price in Common-Value Auctions.' American Economic Review, 82, 512-518

McFadden, D. (1989) 'A Method of Simulated Moments for Estimation of Discrete Response Models Without Numerical Integration.' Econometrica, 57, 995-1026

Milgrom, P. (1985) 'The Economics of Competitive Bidding: A Selective Survey.' In Social Goals and Social Organization, edited by L. Hurwicz, D. Schmeidler, and H. Sonnenschein. (Cambridge: Cambridge University Press)

Milgrom, P. (1987) 'Auction Theory.' In Advances in Economic Theory: Fifth World Congress, edited by T. Bewley. (Cambridge: Cambridge University Press)

Milgrom, P. and R. Weber (1982a) 'A Theory of Auctions and Competitive Bidding.' Econometrica, 50, 1089-1122

Milgrom, P. and R. Weber (1982b) 'The Value of Information in a Sealed Bid Auction.' Journal of Mathematical Economics, 10, 105-114 
Paarsch, H. (1989) 'Empirical Models of Auctions within the Independent Private Values Paradigm and an Application to British Columbian Timber Sales.' Discussion Paper 89-14, Department of Economics, University of British Columbia

Paarsch, H. (1990) 'On Reduced-Form and Structural Empirical Models of Auctions.' Typescript, Department of Economics, University of British Columbia

Paarsch, H. (1991) 'Empirical Models of Auctions and an Application to British Columbian Timber Sales.' Discussion Paper 91-19, Department of Economics, University of British Columbia

Paarsch, H. (1992) 'Deciding between the Common and Private Value Paradigms in Empirical Models of Auctions.' Journal of Econometrics, 51, 191-215

Paarsch, H. (forthcoming) 'A Comparison of Estimators for Empirical Models of Auctions.' Annales d'Economie et de Statistique

Pakes, A. and D. Pollard (1989) 'Simulation and the Asymptotics of Optimization Estimators.' Econometrica, 57, 1027-1057

Perrigne, I., M. Simioni, and Q. Vuong (in progress) 'Nonparametric Estimation of First-Price Auctions.' Institut d'Economie Industrielle, Université des Sciences Sociales de Toulouse

Riley, J. and W. Samuelson (1981) 'Optimal Auctions.' American Economic Review, $71,381-392$

Weverbergh, M. (1979) 'Competitive Bidding with Asymmetric Information Reanalyzed.' Management Science, 25, 291-294

Wilson, R. (1967) 'Competitive Bidding with Asymmetric Information.' Management Science, 13, 816-820 